\title{
Modificações fisiológicas em folhas de pequi (Caryocar brasiliense) causadas pela aplicação de glyphosate ${ }^{1}$
}

\section{Physiological changes in pequi (Caryocar brasiliense) leaves caused by glyphosate application}

\section{Lailla Queiroz Silva ${ }^{2}$; Alana Cristina Ferreira Araújo ${ }^{3}$; Gabriel Martins Almeida ${ }^{4}$; Ailton José Crispim Filho ${ }^{5}$; Alan Carlos Costa ${ }^{6}$; Adriano Jakelaitis ${ }^{7}$}

\begin{abstract}
Resumo - O uso de herbicidas é essencial para o controle de plantas daninhas e aumento da produtividade das culturas, porém esses produtos podem afetar a fisiologia e o crescimento de organismos não alvos, incluindo plantas nativas. Nesta pesquisa, objetivou-se avaliar em casa de vegetação climatizada as trocas gasosas, a fluorescência da clorofila $a$ e o teor de pigmentos cloroplastídicos em plantas de pequi (Caryocar brasiliense) tratadas com glyphosate. Os tratamentos, arranjados em blocos casualizados, corresponderam as doses de 0 (controle), 50, 100, 250, 500, 1.000 e $1.500 \mathrm{~g} \mathrm{ha}^{-1}$ do equivalente ácido de glyphosate, aplicadas aos 120 dias após o transplantio das mudas de pequi. As avaliações de trocas gasosas e de fluorescência da clorofila $a$ foram realizadas as 24,48 e 240 horas após a aplicação (HAA) do herbicida e a de pigmentos cloroplastídicos as 240 HAA. Houve reduções nas taxas fotossintética e transpiratória, na condutância estomática, no rendimento quântico efetivo do fotossistema II, no rendimento quântico potencial do fotossistema II e na taxa aparente de transporte de elétrons, exceto para a relação entre a concentração interna e externa de $\mathrm{CO}_{2}$ e do coeficiente de extinção não fotoquímica, que houve acréscimo em função do aumento das doses de glyphosate. Similarmente, as 240 HAA para as variáveis clorofilas $a, b$, carotenoides e relação clorofila $a / b$ foram observadas reduções lineares nos valores destas variáveis. O glyphosate promove alterações nas variáveis fotossintéticas com reduções nas características de trocas gasosas, na eficiência fotoquímica e no teor de pigmentos cloroplastídicos, principalmente quanto expostas as maiores doses do herbicida.

Palavras-chaves: fluorescência da clorofila $a$; herbicida; pigmentos cloroplastídicos; trocas gasosas
\end{abstract}

\footnotetext{
Abstract - The use of herbicides is essential for the control of weeds and increase crop productivity, but these products can affect the physiology and growth of non-target organisms, including native plants. The objective of this research was to evaluate in the heated greenhouse the gas exchange, fluorescence of chlorophyll $a$ and chloroplastid pigment content of pequi plants (Caryocar brasiliense) submitted to simulated drift of glyphosate. The treatments were arranged

${ }^{1}$ Recebido para publicação em 09/05/2016 e aceito em 10/06/2016.

${ }^{2}$ Mestre em Ciências Agrárias - Agronomia (PPGCA-Agro), Instituto Federal Goiano (IF Goiano), Caixa Postal, CEP: 75901-970, Rio Verde, Goiás, Brasil. E-mail: <lailla.queiroz@ hotmail.com>.

${ }^{3}$ Acadêmica do curso de Agronomia, IF Goiano, Rio Verde, Goiás, Brasil. E-mail: <alanashego@ hotmail.com>.

${ }^{4}$ Acadêmico do curso de Agronomia, IF Goiano, Rio Verde, Goiás, Brasil. E-mail: <gabrielrvmartins@ gmail.com>.

${ }^{5}$ Acadêmico do curso de Agronomia, IF Goiano, Rio Verde, Goiás, Brasil. E-mail: <ailtonfilho_@ hotmail.com>.

${ }^{6}$ Professor do PPGCA-Agro, IF Goiano, Rio Verde, Goiás, Brasil. E-mail: <alcarcos@ gmail.com>.

${ }^{7}$ Professor do PPGCA-Agro, IF Goiano, Rio Verde, Goiás, Brasil. E-mail: <ajakelaitis@ yahoo.com.br>.
} 
in a randomized block. Seven doses of glyphosate were tested: 0 (control), 50, 100, 250, 500, 1,000 and $1,500 \mathrm{~g} \mathrm{ha}^{-1}$ of the acid equivalent. The herbicide was applied at 120 days after transplanting the seedlings. Evaluations of gas exchange and chlorophyll fluorescence were taken 24, 48 and 240 hours after application (HAA) of the herbicide. The chloroplastid pigments was evaluated the 240 HAA. There were reductions in photosynthetic and transpiration rates, stomatal conductance, the effective quantum yield of photosystem II, in the potential quantum yield of photosystem II and apparent electron transport rate, except for the relationship between internal and external $\mathrm{CO} 2$ concentration and extinction coefficient photochemistry not that there was an increase, due to the increase of the glyphosate doses. Similarly, the 240 HAA were observed reductions linear in the values of the variables chlorophyll $a, b$, carotenoids and relation chlorophyll a/b. Glyphosate causes changes in photosynthetic variables with reductions in the characteristics of gas exchange, photochemical efficiency and chloroplastid pigment content, especially as exposed to higher doses of the herbicide.

Keywords: chlorophyll $a$ fluorescence; herbicide; chloroplastid pigments; gas exchange

\section{Introdução}

Caryocar brasiliense Camb. (pequi) é uma árvore originária do Brasil e considerada símbolo do bioma Cerrado. Neste bioma, encontra-se grandes plantações naturais de pequi principalmente nos estados de Minas Gerais, São Paulo, norte do Paraná, Goiás, Mato Grosso, Tocantins, sul da Bahia, sul do Pará e sul do Maranhão (Macedo, 2005; Kerr et al., 2007). A exploração dos frutos é realizada por meio extrativista e constitui-se em importante fonte de renda familiar da comunidade rural.

Entretanto, as populações naturais do pequizeiro têm sido comprometidas devido a constante e intensa exploração do Cerrado brasileiro. As principais ameaças à biodiversidade no Cerrado estão na expansão da agricultura e da pecuária que se consolidaram com uso significativo de fertilizantes químicos, intensa mecanização e emprego de alta tecnologia com agrotóxicos, dentre estes, os herbicidas (Cunha et al., 2008).

Dentre os herbicidas mais usados no Brasil, o ghyphosate tem-se destacado pelas suas características físico-químicas e econômicas e pela eficácia agronômica no controle de elevado número de espécies de plantas daninhas. Seu mecanismo de ação está ligado à inibição da enzima EPSPs (5enolpiruvilchiquimato-3-fosfato sintase), necessária na via do ácido chiquímico, que é usada na síntese de vários metabólitos das plantas (Nandula et al., 2007). Todavia seu uso tem causado sérios problemas ambientais devido à deposição do produto fora do alvo de interesse (França et al., 2010; Pereira et al., 2010; Tiburcio et al., 2012).

Como exemplo, a deriva de herbicidas causa prejuízos em fragmentos florestais e também em culturas adjacentes não-alvos, sendo de grande importância a identificação dos danos (Boutin et al., 2014). A sintomatologia e os danos em plantas não-alvo dependem de diversos fatores como a espécie, o estádio de desenvolvimento da planta, o clima e a quantidade de princípio ativo que chega à planta receptora (Rigoli et al., 2008; Pereira et al., 2010).

Pesquisas com deriva simulada de herbicidas têm permitido a avaliação da ocorrência de injúrias e de modificações nos padrões de crescimento vegetal pela ação do glyphosate (Tuffi Santos et al., 2009; Machado et al., 2010). Os principais sintomas visuais observados após a exposição são a clorose, seguida de necrose e morte da planta (França et al., 2010). Contudo, uma importante ferramenta que auxilia precocemente na determinação dos danos provocados por herbicidas são as avaliações fisiológicas da folha. Herbicidas geralmente provocam efeitos totalmente nocivos à fotossíntese e variáveis relacionadas a fotossíntese como condutância estomática e taxa 
fotossintética (Carvalho, 2011), taxa transpiratória (Pereira et al., 2010), fluorescência da clorofila $a$ (Ferreira et al., 2015) e o teor de pigmentos cloroplastídeos (Santos et al., 1999; Meschede et al., 2011) são afetadas pelo glyphosate.

A redução na produtividade da planta pode ser consequência da redução da taxa fotossintética, dependendo da intensidade de intoxicação provocado pelo herbicida. Nesta pesquisa objetivou-se avaliar se as trocas gasosas, a fluorescência da clorofila $a$ e o teor de pigmentos cloroplastídicos são afetadas quando plantas de pequi são tratadas com diferentes doses de glyphosate.

\section{Material e Métodos}

Esta pesquisa foi conduzida em condições de casa de vegetação climatizada no período de fevereiro a julho de 2014. As mudas de Caryocar brasiliense Camb. foram obtidas em viveiro procedente da cidade de Guapó-GO. Foram selecionadas e adquiridas mudas com altura média de $30 \mathrm{~cm}$ e posteriormente foram transplantadas para vasos com volume de 18 litros.

Para o preenchimento dos vasos empregou-se terra de subsolo, da qual a análise química foi constituída de: $\mathrm{pH}$ em $\mathrm{CaCl}_{2}$ de 5,6; $22,84 \mathrm{mg} \mathrm{dm}^{-3}$ de P;190 $\mathrm{mg} \mathrm{dm}^{-3}$ de $\mathrm{K} ; 5,98$ $\mathrm{cmol}_{\mathrm{c}} \mathrm{dm}^{-3}$ de Ca; $1,80 \mathrm{cmol}_{\mathrm{c}} \mathrm{dm}^{-3}$ de $\mathrm{Mg} ; 2,80$ $\mathrm{cmol}_{\mathrm{c}} \mathrm{dm}^{-3}$ de $\mathrm{H}+\mathrm{Al} ; 2,45 \mathrm{dag} \mathrm{kg}^{-1}$ de matéria orgânica e $74,7 \%$ de saturação por bases.

Para fertilização em cada vaso foram utilizados $100 \mathrm{~g}$ de superfosfato simples e $20 \mathrm{~g}$ de cloreto de potássio. Após o transplante da muda, a cada 30 dias, foram utilizados $2 \mathrm{~g} \mathrm{vaso}^{-}$ ${ }^{1}$ de fertilizante, contendo em porcentagem $\mathrm{N}$ : 13; $\mathrm{P}_{2} \mathrm{O}_{5}: 5 ; \mathrm{K}_{2} \mathrm{O}: 13 ; \mathrm{B}: 0,04 ; \mathrm{Ca}: 1 ; \mathrm{Cu}: 0,05$; S: 5; Fe: 0,2; Mg: 1; Mn: 0,08; Mo: 0,005; e Zn: 0,15 .

Após 120 dias do transplantio, as mudas foram separadas por tamanho para a padronização dos blocos. Os tratamentos corresponderam à deriva simulada de seis doses de $\left(50,100,250,500,1.000\right.$ e 1.500 g e.a. ha $\left.{ }^{-1}\right)$ e um tratamento sem herbicida (controle) no delineamento de blocos casualizados com nove repetições. A unidade experimental foi de um vaso contendo uma planta de pequi.

A deriva simulada consistiu da aplicação do herbicida glyphosate (Roundup Transorb ${ }^{\circledR}$, 480 g e.a. $\mathrm{L}^{-1}$, SC, Monsanto) utilizando um pulverizador costal com pressão constante mantida por $\mathrm{CO}_{2}$ comprimido, munido de barra com quatro pontas de pulverização e bico da série XRTeejet ${ }^{\circledR}$, tipo leque modelo XR11002VP. Aplicou-se o equivalente a $120 \mathrm{~L} \mathrm{ha}^{-1}$ de calda. As plantas foram colocadas fora da casa de vegetação e no momento da aplicação a temperatura máxima foi de $18,3{ }^{\circ} \mathrm{C}$, a velocidade do vento de $2,9 \mathrm{~m} \mathrm{~s}^{-1}$ e umidade relativa de $90 \%$. Após a aplicação do herbicida os vasos foram transferidos para o interior da casa de vegetação. Durante todo o período experimental, a temperatura no interior da casa de vegetação variou entre 22 a $29{ }^{\circ} \mathrm{C}$ e a umidade relativa entre 65 a $75 \%$.

Foram avaliadas as trocas gasosas das plantas de $C$. brasiliense para registro das taxas

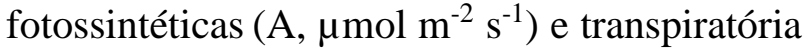
(E, mmol m $\mathrm{m}^{-2} \mathrm{~s}^{-1}$ ), da condutância estomática (gs, mol $\mathrm{H}_{2} \mathrm{O} \mathrm{m} \mathrm{m}^{-2} \mathrm{~s}^{-1}$ ) e da relação entre a concentração interna e externa de $\mathrm{CO}_{2}(\mathrm{Ci} / \mathrm{Ca})$. Estas avaliações foram realizadas utilizando um analisador automático de fotossíntese modelo LI-6400XTR (Licor $^{\circledR}$, Nebrasca, Estados Unidos) com temperatura do bloco de $24{ }^{\circ} \mathrm{C}$ e densidade de fluxo de fótons igual a $1000 \mu \mathrm{mol}$ $\mathrm{m}^{-2} \mathrm{~s}^{-1}$. As avaliações foram realizadas aos 24, 48 e 240 horas após a aplicação (HAA) do glyphosate e especificamente entre as 08:30 e 10:30 horas. As avaliações foram feitas no terceiro par de folhas totalmente expandidas, e em cada folha foi utilizada sempre a mesma área.

A fluorescência da clorofila $a$ foi avaliada também no terceiro par de folhas totalmente expandidas, paralelamente às medições de trocas gasosas, utilizando-se de um fluorômetro portátil modelo MINI-PAM (Walz, Effeltrich, Alemanha), munido de pinça específica para suporte da folha (modelo 2030- 
B) (Rascher et al., 2000). Inicialmente, as folhas foram adaptadas ao escuro por 30 minutos e após este período os tecidos foliares foram expostos a uma luz vermelha modulada $(0,03$ $\left.\mu \mathrm{mol} \mathrm{m} \mathrm{m}^{-2} \mathrm{~s}^{-1}\right)$ e determinou-se a fluorescência inicial $\left(\mathrm{F}_{0}\right)$. Posteriormente, foi feita aplicação de um pulso de $0,8 \mathrm{~s}$ de luz actínica saturante

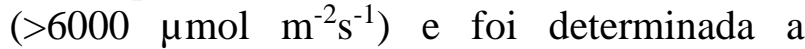
fluorescência máxima (Fm). Com estes resultados, estimou-se o rendimento quântico potencial do fotossistema II, utilizando a equação $\mathrm{Fv} / \mathrm{Fm}=\left(\mathrm{Fm}-\mathrm{F}_{0}\right) / \mathrm{Fm}$.

$\mathrm{O}$ rendimento quântico efetivo do fotossistema II foi determinado por meio da sobreposição de um pulso de saturação em folhas previamente adaptadas à luz ambiente, sendo calculado como $\Delta \mathrm{F} / \mathrm{Fm}^{\prime}=\left(\mathrm{Fm}^{\prime}-\mathrm{F}\right) / \mathrm{Fm}$ ', em que $\mathrm{F}$ é o rendimento da fluorescência máxima durante o pulso de saturação (GENTY et al., 1989). O $\Delta \mathrm{F} / \mathrm{Fm}$ ' foi utilizado para estimar a taxa aparente de transporte de elétrons (ETR), mediante o uso da equação $\mathrm{ETR}=\Delta \mathrm{F} / \mathrm{Fm} \mathrm{xDFFx}^{\mathrm{x}}$,5x0,84, em que DFF é a densidade de fluxo de fótons ( $\mu$ mol $\mathrm{m}^{-2} \mathrm{~s}^{-1}$ ) incidente sobre a folha; 0,5 é o valor correspondente à fração da energia de excitação distribuída para o FSII; e 0,84 é o valor correspondente à fração de luz incidente absorvida pelas folhas.

O coeficiente de extinção não fotoquímica de Stern-Volmer foi calculado como $\left.\mathrm{NPQ}=(\mathrm{Fm}-\mathrm{Fm})^{\prime}\right) / \mathrm{Fm}$ ', (Bilger e Bjorkman, 1990). Da mesma forma descrita para as trocas gasosas, as avaliações com o fluorômetro portátil foram realizadas entre as 08:30 e 10:30 horas, sempre na mesma área de cada folha em avaliações realizadas aos 24, 48 e 240 HAA.

Determinou-se também o teor de pigmentos cloroplastídicos, coletando-se de cada unidade experimental, três discos foliares de folhas totalmente expandidas do terço médio da planta. As coletas foram realizadas as 240 horas após a aplicação do glyphosate. Os discos foram colocados em frascos de vidro protegidos da luz, com $5 \mathrm{~mL}$ de dimetilsulfóxido (DMSO).
Em seguida, os frascos foram levados ao banho-maria à temperatura de $65^{\circ} \mathrm{C}$ por 24 horas. Após este período foram realizadas as leituras em espectrofotômetro (Evolution 60S, Thermo Fisher Scientific Inc., MA, Estados Unidos) nas absorbâncias de $480 \mathrm{~nm}$ para quantificação dos carotenoides, $649 \mathrm{~nm}$ para clorofila $b$ e $665 \mathrm{~nm}$ para clorofila $a$.

Os resultados foram submetidos à análise de variância (ANOVA) pelo teste $\mathrm{F}$ e ajustados aos modelos de regressão, adotando o nível de significância de 5\%. Os modelos foram escolhidos pela simplicidade, significado biológico e pelo coeficiente de determinação. As análises estatísticas foram realizadas por meio do programa estatístico Assistat (versão 7.7 beta 2014) e pelo software Sigmaplot V.12 (SPSS Inc., Estados Unidos).

\section{Resultados e Discussão}

Após a aplicação do glyphosate foram observados efeitos significativos nas plantas de pequi para as variáveis fisiológicas relativas à taxa fotossintética (Figura 1A), a condutância estomática (Figura 1B), a taxa transpiratória (Figura 2A) e a relação entre a concentração interna e externa de $\mathrm{CO}_{2}$ (Figura 2B) nas avaliações realizadas as 24, 48 e 240 HAA.

Para a taxa fotossintética os resultados se ajustaram a modelos lineares, que comprovaram os efeitos negativos do glyphosate sobre as plantas de pequi em função das doses aplicadas, com queda de 55,49 e $63 \%$ as 24,48 e 240 HAA, respectivamente, da maior dose $(1.500 \mathrm{~g}$ e.a. ha $^{-1}$ ), se comparada ao controle. Contudo, entre as três curvas obtidas maior coeficiente angular foi observado para a avaliação realizada as 240 HAA com queda de $0,0045 \mu \mathrm{mol} \mathrm{m}^{-2} \mathrm{~s}^{-1}$ para cada grama de equivalente ácido do glyphosate aspergido sobre as plantas (Figura 1A).

Da mesma forma para a condutância estomática do pequi os resultados se ajustaram a modelos lineares e a medida que se aumentaram as doses de glyphosate ocorreram reduções de $68 \%$ no primeiro dia após a aplicação, de $53 \%$ 
as $48 \mathrm{HAA}$ e de $75 \%$ as $240 \mathrm{HAA}$ da maior dose (1.500 g e.a. ha $\left.{ }^{-1}\right)$, se comparadas ao controle (Figura 1B). Para a taxa transpiratória, os efeitos observados foram similares à taxa fotossintética e de condutância estomática para todas as épocas avaliadas com efeitos decrescentes com o aumento das doses do herbicida (Figura 2A) sendo mais pronunciada as 240 HAA, com redução de $68 \%$ para a maior dose de herbicida em relação ao controle.
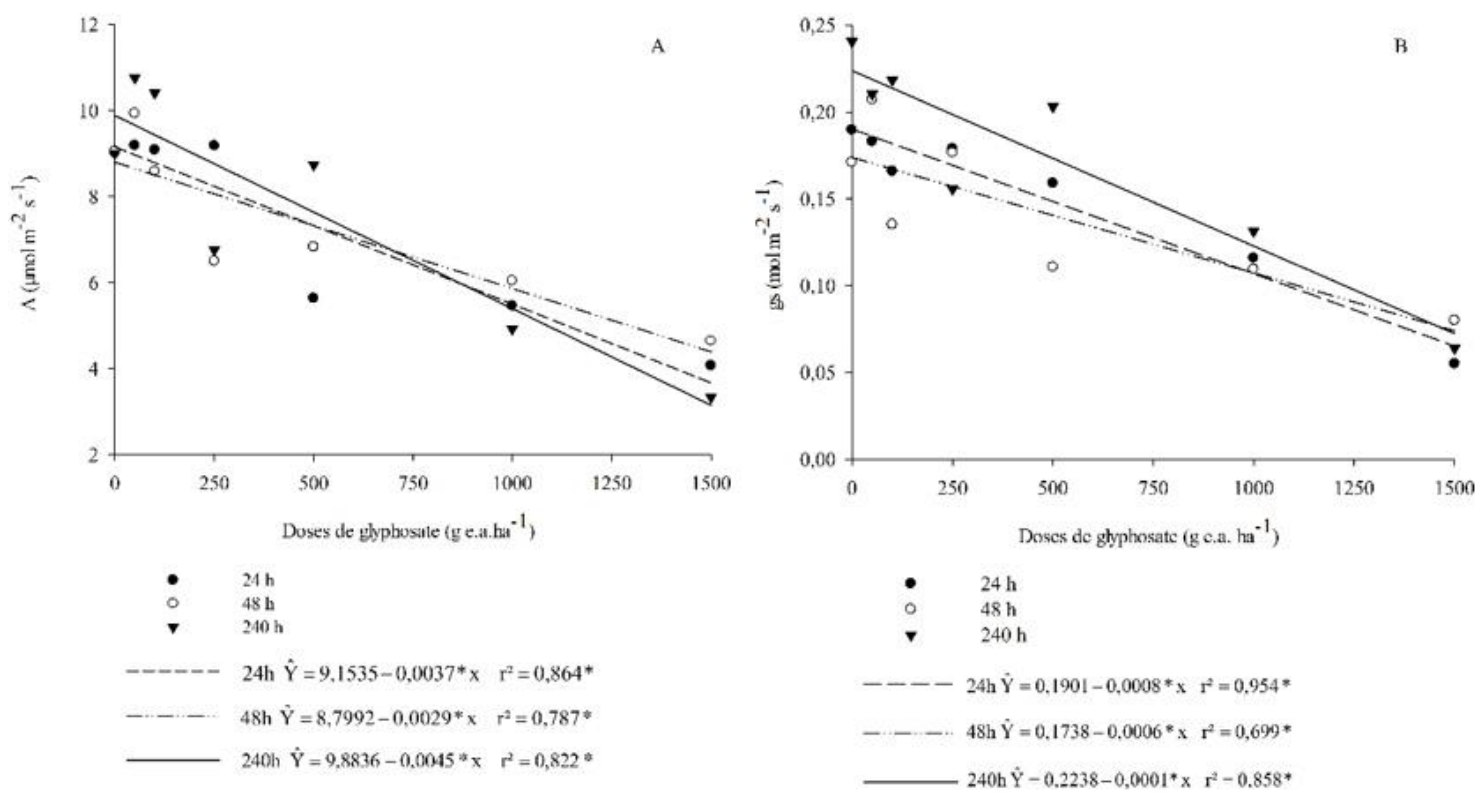

Figura 1. Taxa fotossintética (A) (A em $\left.\mu \mathrm{mol} \mathrm{m} \mathrm{m}^{-2} \mathrm{~s}^{-1}\right)$ e condutância estomática (B) ( $g s$ em mol m $\mathrm{m}^{-}$ $2 \mathrm{~s}^{-1}$ ) de plantas de pequi submetidas à deriva simulada de glyphosate e avaliadas as 24,48 e 240 horas após a aplicação. Rio Verde (GO), 2014.
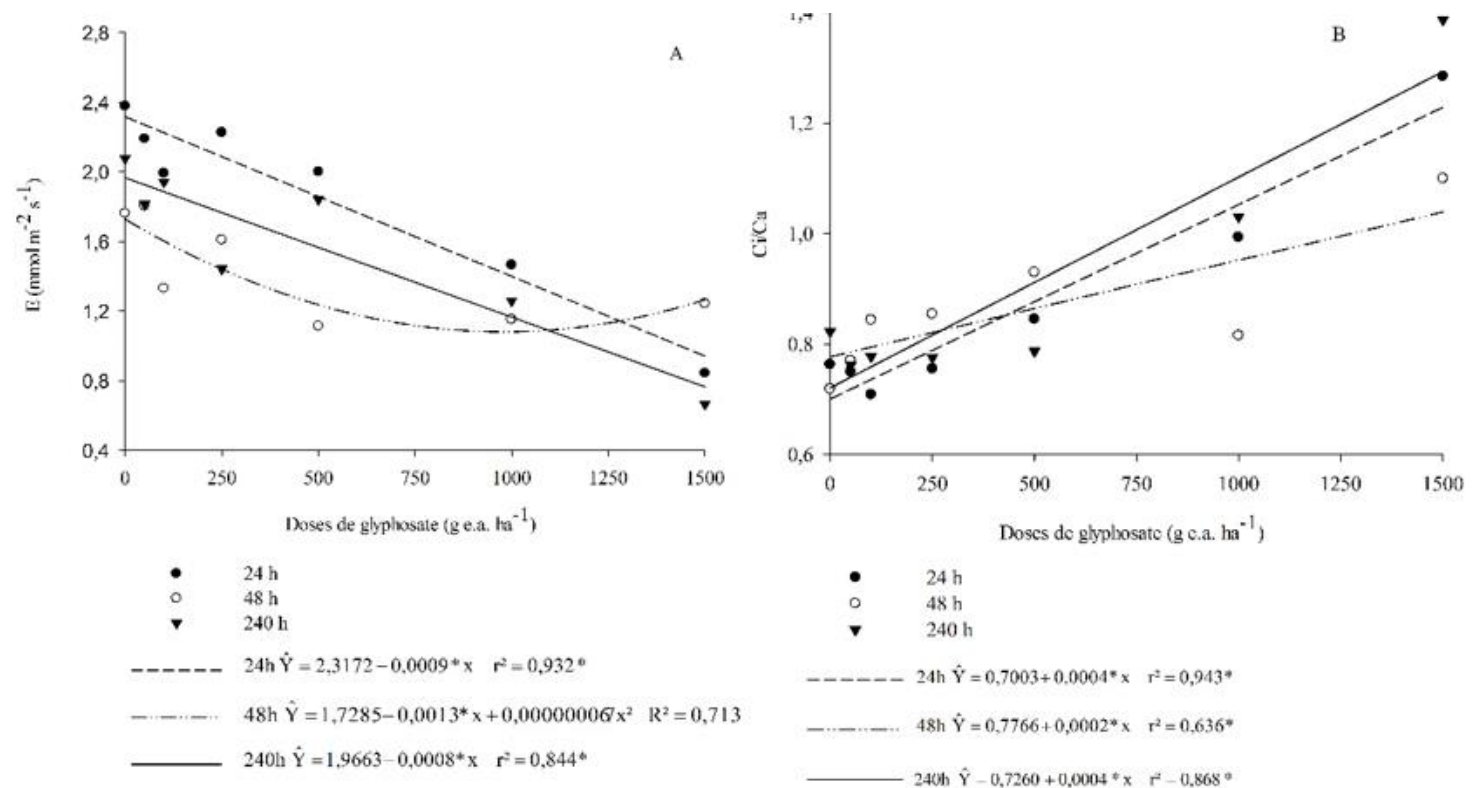

Figura 2. Taxa transpiratória (A) $\left(E\right.$ em mmol m $\left.\mathrm{m}^{-2} \mathrm{~s}^{-1}\right)$ e relação entre a concentração interna e externa $(\mathrm{B})(\mathrm{Ci} / \mathrm{Ca})$ de plantas de pequi submetidas à deriva simulada de glyphosate e avaliadas as 24, 48 e 240 horas após a aplicação. Rio Verde (GO), 2014. 
A relação $\mathrm{Ci} / \mathrm{Ca}$ também foi afetada pelas doses do produto havendo aumento da concentração interna de $\mathrm{CO}_{2}$ em função do aumento das doses do herbicida. Os modelos que descrevem a resposta $(\mathrm{Ci} / \mathrm{Ca})$ se ajustaram a modelos lineares e caracterizou-se por aumento de $70 \%$ as 24 e 240 HAA, e de $53 \%$ as 48 HAA com relação a maior dose e o controle (Figura 2B).

O uso incorreto de herbicidas tem promovido a contaminação ambiental e de alimentos, a intoxicação de pessoas e os problemas associados as próprias práticas agrícolas como a intoxicação de culturas subsequentes (carryover) e o surgimento de plantas daninhas resistentes aos herbicidas. No entanto, são escassos na literatura trabalhos que relacionam o impacto dos herbicidas na biodiversidade vegetal do entorno das áreas agrícolas, principalmente quando estes são carreados pelo vento durante a aplicação.

Embora a fotossíntese não seja afetada diretamente pela ação do glyphosate, diversos trabalhos relatam alterações no metabolismo do carbono (Machado et al., 2010; Zobiole et al., 2010a, 2010b), os quais são confirmados neste estudo pelas alterações provocadas nas trocas gasosas de plantas de pequi, evidenciados pelas reduções na taxa fotossintética, condutância estomática, taxa transpiratória e pelo aumento na relação Ci/Ca. Batista (2014) evidenciou para o glyphosate em pesquisa com a espécie Pouteria torta (guapeva) que o ácido chiquímico juntamente com a condutância estomática são bons biomarcadores da ação do herbicida.

Essas alterações podem estar relacionadas com a desregulação da abertura e do fechamento estomático que é um importante fator que contribui na assimilação de $\mathrm{CO}_{2}$. Como a fotossíntese depende do seu constante fluxo e a condutância estomática é responsável pelo fluxo de gases nos estômatos; assim, quanto menor for à sua abertura maior a resistência estomática e a sua redução provoca efeitos significativos na taxa fotossintética e transpiratória, provocando a redução da eficiência carboxilativa (Machado et al., 2010; Zobiole et al., 2010a). Estes efeitos foram observados na redução da condutância estomática já no primeiro dia após a aplicação (24 HAA) e consequentemente as reduções na taxa fotossintética e taxa transpiratória que se mantiveram até as 240 HAA (Figuras 1A, 1B, 2A). Da mesma forma, Vital (2015) pesquisando plantas de girassol tratadas com subdoses do glyphosate observaram acúmulo de $\mathrm{CO}_{2}$ na câmara subestomática a partir de 24 horas da aplicação do herbicida, indicando danos no metabolismo fotossintético destas plantas.

A eficiência carboxilativa pode ser diminuída tanto pelo fornecimento de energia (ATP) e de poder redutor (NADPH) à fase bioquímica, quanto pela inibição de enzimas do ciclo de Calvin, conforme verificado com a Rubisco que é inibida em plantas tratadas com glyphosate (Ahsan et al., 2008). Os resultados encontrados também corroboram com Zobiole et al. (2010b) que trabalhando com soja geneticamente modificada para resistência ao glyphosate (RR) e com diferentes doses aplicadas do herbicida (600, 900, 1200, 1800 e $2400 \mathrm{~g} \mathrm{e}^{\mathrm{a}} \mathrm{ha}^{-1}$ ) verificaram que a condutância estomática, taxa fotossintética e taxa transpiratória foram reduzidas e houve acréscimo na relação $\mathrm{Ci} / \mathrm{Ca}$ com o aumento das doses. Os dados também são confirmados por Machado et al. (2010) que avaliando diferentes doses de glyphosate $(43,2 ; 86,2 ; 129,6$ e $172,8 \mathrm{~g}$ $\mathrm{ha}^{-1}$ ) em clones de eucalipto constataram que aos 21 dias após a aplicação (DAA), com o incremento da dose de glyphosate houve uma redução na condutância estomática, taxa fotossintética e taxa transpiratória.

Observaram-se efeitos das doses de glyphosate nas variáveis concernentes a fluorescência da clorofila $a$ observadas nas diferentes épocas de avaliação após a aplicação do glyphosate (Figuras 3 e 4). As 240 HAA nas doses acima de 500 g e.a. ha ${ }^{-1}$ o herbicida promoveu danos no fotossistema II (Figura 3A) com redução de $15 \%$ em relação a maior dose e o controle. Para as demais avaliações do 
rendimento quântico máximo do FSII (Fv/Fm) os valores ficaram entre 0,81 e 0,82 para 24 e 48 HAA, respectivamente (Figura 3A).

Para o rendimento quântico efetivo do FSII $(\Delta \mathrm{F} / \mathrm{Fm}$ ') e a ETR houve um comportamento semelhante manifestado pela redução destas variáveis em função do aumento das doses de glyphosate, contudo, não foram possíveis o ajuste de modelos de regressão linear e não linear, sendo os resultados

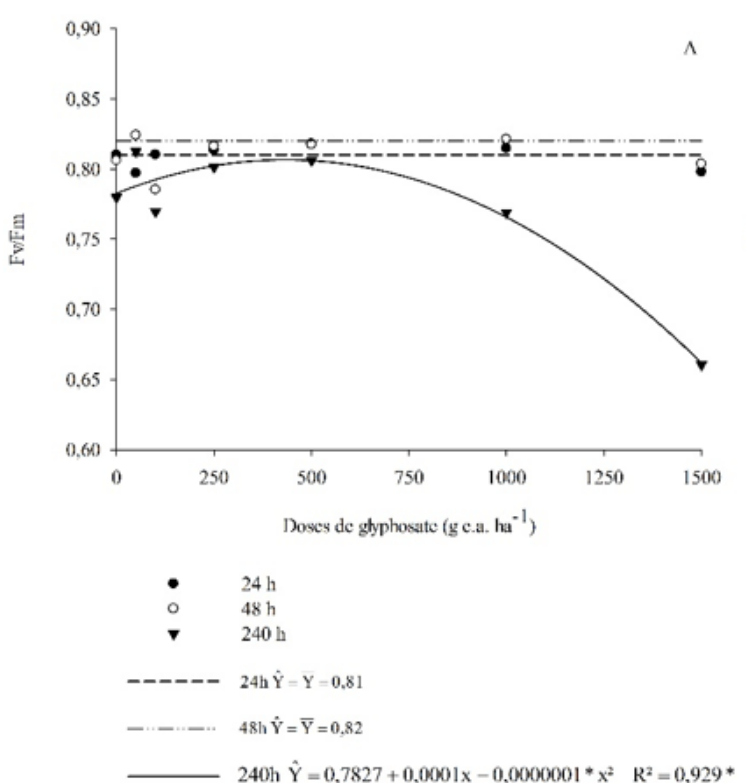

representados por gráficos de linhas (Figuras 3B e 4A). Foram observadas para ambas as variáveis, que as 240 HAA nas doses de 250 e $500 \mathrm{~g}$ e.a. ha ${ }^{-1}$ houve uma possível recuperação da planta, e em doses superiores queda expressiva nestes valores. Já para o NPQ (Figura 4B) os resultados se ajustaram ao modelo quadrático e foi observado acréscimo dos valores com o aumento das doses principalmente as 240 HAA.

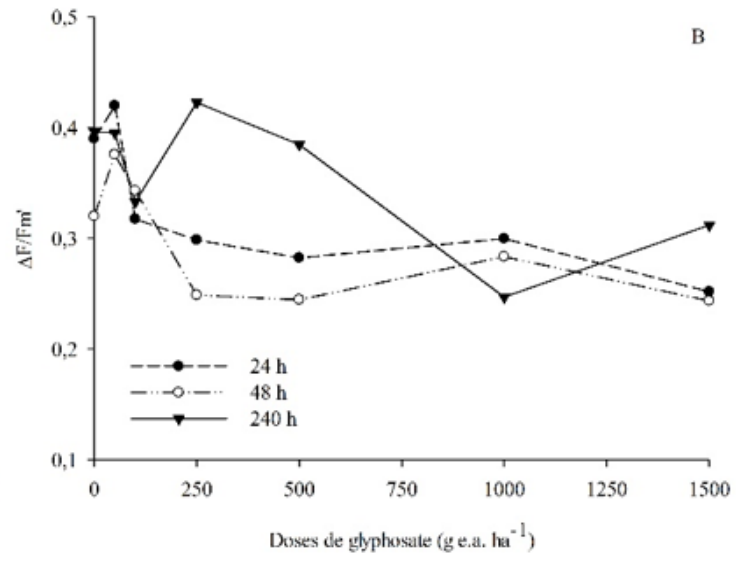

Figura 3. Rendimento quântico máximo (A) (Fv/Fm) e rendimento quântico efetivo do FSII (B) $(\Delta \mathrm{F} / \mathrm{Fm}$ ') de plantas de pequi submetidas à deriva simulada de glyphosate e avaliadas as $24,48 \mathrm{e}$ 240 horas após a aplicação. Rio Verde (GO), 2014.

A fluorescência da clorofila $a$ é uma medida da eficiência fotossintética e a produtividade da planta, podendo ser usada como uma ferramenta no estudo de vários aspectos fotossintéticos refletindo alterações na organização da membrana dos tilacóides (Zobiole et al., 2010b). Valores de referência para Fv/Fm quando a planta está com seu aparelho fotossintético intacto, varia entre 0,75 e 0,85, e as plantas de pequi aos 240 HAA apresentaram valores abaixo dos de referência indicando danos ao aparato fotossintético (Wagner e Merotto Junior, 2014). Por outro lado, a diminuição nos valores de $\Delta \mathrm{F} / \mathrm{Fm}$ ' refletiu na redução da eficiência de captura de energia de excitação pelos centros de reações abertos do FSII, corroborando com as observações dos menores valores de ETR encontrados nas menores doses (Lu et al., 2003).

Resultados semelhantes também foram observados por Zobiole et al. (2010a) que em pesquisas com soja geneticamente modificada para resistência ao glyphosate tratadas com as doses de 800, 1.200 e 2.400 g e.a. ha ${ }^{-1}$, verificaram que os parâmetros da fluorescência da clorofila $a$ foram afetados pelo glyphosate, e que houve uma diminuição linear da condutância estomática e da ETR com o aumento das doses. O aumento do NPQ está indicando que o glyphosate promoveu danos às 
plantas de pequi e que elas estão dissipando mais energia na forma de calor (Taiz e Zeiger, 2009). Este aumento na dissipação não fotoquímica na forma de calor representada pelos altos valores de NPQ, como importante mecanismo de fotoproteção, ficou evidenciado nas maiores doses de glyphosate, particularmente acima de $500 \mathrm{~g}$ e.a. $\mathrm{ha}^{-1}$ as 240 HAA.

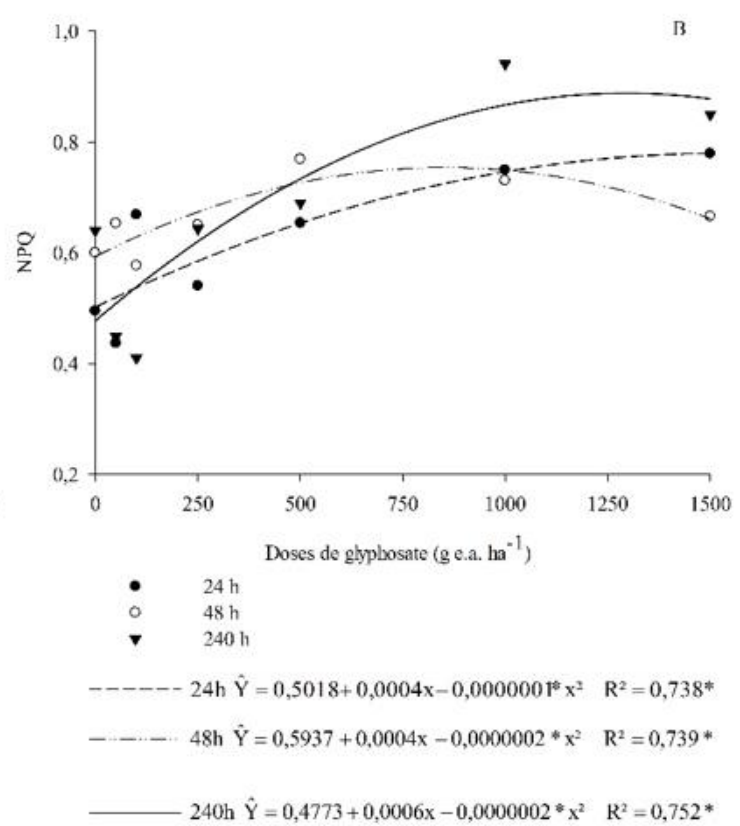

Figura 4. Taxa de transporte de elétrons (A) (ETR) e quenching não fotoquímico (B) (NPQ) de plantas de pequi submetidas à deriva simulada de glyphosate e avaliadas as 24, 48 e 240 horas após a aplicação. Rio Verde (GO), 2014.

O aumento das doses de glyphosate contribuiu para o aumento da degradação de clorofilas $a$ e $b$, de carotenoides e da relação entre clorofila $a$ e $b$ (Figuras 5 e 6), sendo o comportamento explicado por modelos lineares simples. As 240 HAA nas doses acima de $500 \mathrm{~g}$ e.a. ha $^{-1}$ o herbicida reduziu em 28,$7 ; 27,5$ e $35,1 \%$ no teor de clorofila a (Figura 5A), clorofila $b$ (Figura 5B) e carotenoides (Figura $6 \mathrm{~A})$ respectivamente, em relação a maior dose $\mathrm{e}$ o controle, com queda de 0,0072, 0,0022 e $0,0032 \mu \mathrm{g} \mathrm{cm}^{-2}$ para cada grama de equivalente ácido de glyphosate aplicado, respectivamente.

Associado com a redução das trocas gasosas e da fluorescência da clorofila $a$ o aumento da exposição ao glyphosate reduziu a concentração de clorofilas e de carotenoides o que reduz o tamanho do complexo antena e a redução na captação e transferência de energia para os fotossistemas, o que é comprovado com a redução da ETR observado também com o aumento das doses de glyphosate (Figura 4A). Santos et al. (1999) também observaram que o herbicida glyphosate causou um decréscimo nos pigmentos clorofilianos de Spirodela punctata em concentrações mais elevadas $(0,5$ e $5,0 \mathrm{mg}$ $\left.\mathrm{L}^{-1}\right)$, bem como na relação clorofila $a / b$.

De acordo com Santos et al. (1999) a redução da razão clorofila a/b é uma indicação de que as folhas estão em processo de envelhecimento e que diminuição da razão clorofila a/b pelo glyphosate pode indicar que o herbicida tenha intensificado a senescência foliar. Meschede et al. (2011) avaliando as alterações fisiológicas em cana-de-açúcar pela aplicação de glyphosate observaram nas maiores doses testadas (200 e $400 \mathrm{~mL} \mathrm{ha}^{-1}$ ) interferência na fotossíntese por diminuir os 
teores de clorofila e carotenoides, mostrando que estas variáveis são indicadoras do estado de fitointoxicação da planta.

Em síntese, destaca-se pelos resultados obtidos que o glyphosate promove modificações no aparato fotossintético de plantas de pequi, porém alterações significativas na fisiologia da planta ocorrem em doses superiores a $250 \mathrm{~g} \mathrm{ha}^{-}$
${ }^{1}$ de equivalente ácido aspergido, e que em doses menores nota-se a tolerância da planta em relação ao herbicida. Contudo, o biomonitoramento passivo em condições naturais com plantas de pequizeiro localizadas em fragmentos florestais adjacentes às lavouras e sujeitas à deriva deste herbicida se fazem necessários para confirmação dos resultados.
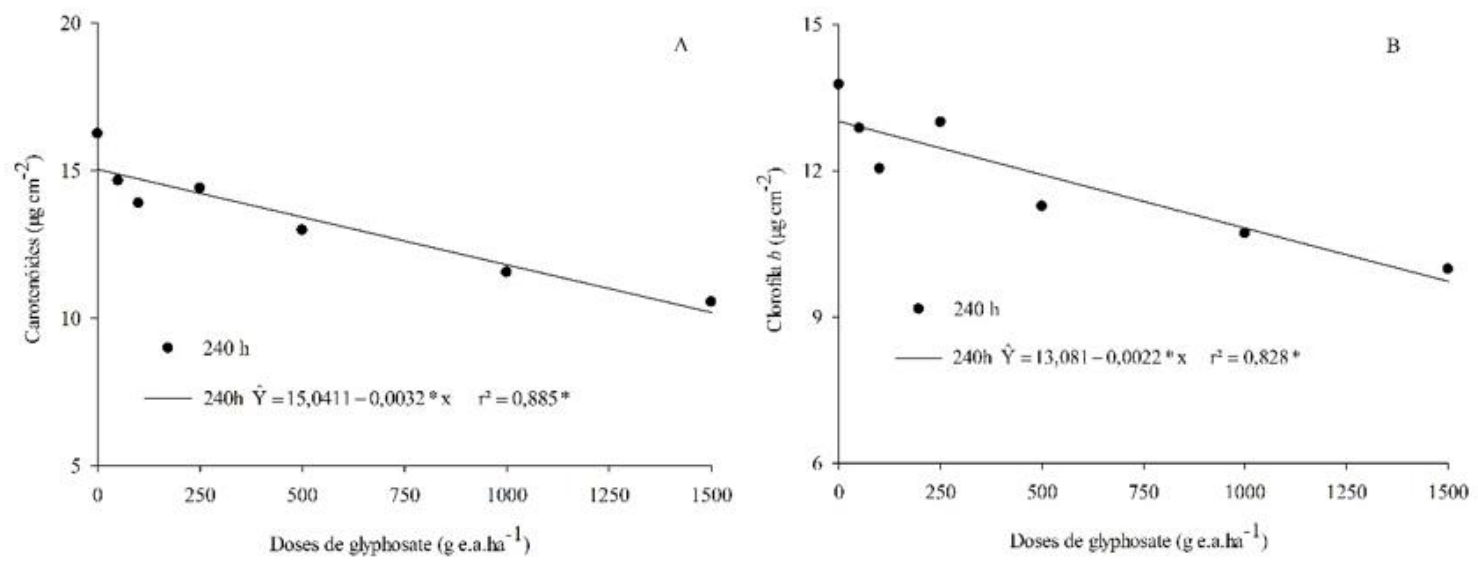

Figura 5. Clorofila $a(\mathrm{~A})$ e $b(\mathrm{~B})\left(\mu \mathrm{g} \mathrm{cm}^{-2}\right)$ de plantas de pequi submetidas à deriva simulada de glyphosate e avaliadas as 240 horas após a aplicação. Rio Verde (GO), 2014.
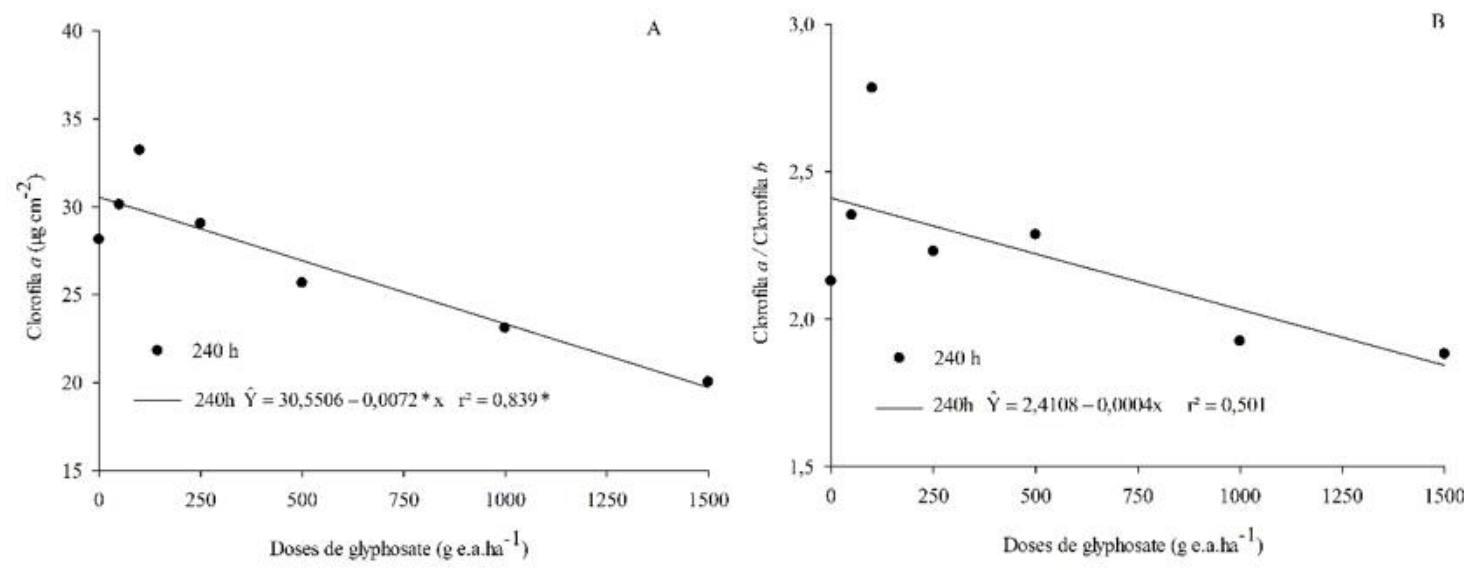

Figura 6. Carotenoides (A) $\left(\mu \mathrm{g} \mathrm{cm}^{-2}\right)$ e relação clorofila $a$ e $b(\mathrm{~B})$ de plantas de pequi submetidas à deriva simulada de glyphosate e avaliadas as 240 horas após a aplicação. Rio Verde (GO), 2014.

\section{Conclusões}

O glyphosate promove alterações nas variáveis fotossintéticas de plantas de pequi com reduções nas características de trocas gasosas, na eficiência fotoquímica e no teor de pigmentos cloroplastídicos, principalmente quanto expostas as maiores doses do herbicida.

\section{Agradecimentos}

Ao $\mathrm{CNPq}$ pelo auxílio financeiro por meio da Chamada Pública MCT/CNPq/MEC/CAPES - Ação Transversal no 06/2011 - Casadinho/Procad e à FINEP pela Chamada Pública MCT/FINEP/CT-INFRA PROINFRA - 02/2010. 


\section{Referências}

Ahsan, N.; Lee, D.G.; Lee, K.W.; Alam, I.; Lee, S.H.; Bahk, J.D.; Byung-Hyun, L. Glyphosateinduced oxidative stress in rice leaves revealed by proteomic approach. Plant Physiology and Biochemistry, v.46, n.12, p.1062-1070, 2008.

Batista, P.F. Biomarcadores da ação do glyphosate em plantas Pouteria torta (mart.) radlk: uma espécie nativa do domínio Cerrado. 2014. 65 f. Dissertação (Mestrado em Ciências Agrárias - Agronomia). IF Goiano Campus-Rio Verde, Rio Verde, 2014.

Boutin, C.; Strandberg, B.; Carpenter, D.; Mathiassen, S.K.; Thomas, P.J. Herbicide impact on non-target plant reproduction: What are the toxicological and ecological implications? Environmental Pollution, v.185, p.295-306, 2014.

Carvalho, F.P. Características fisiológicas do cafeeiro após aplicação do glyphosate. 2011. 60 f. Dissertação (Mestrado em Produção Vegetal) - Universidade Federal dos Vales do Jequitinhonha e Mucuri, Diamantina, 2011.

Cunha, N.R.S.; Lima, J.E.; Gomes, M.F.M.; Braga, M.J. A intensidade da exploração agropecuária como indicador da degradação ambiental na região dos Cerrados, Brasil. Piracicaba/SP. Revista de Economia e Sociologia Rural, v.46, n.2, p.291-323, 2008.

Ferreira, E.A.; Matos, C.C.; Barbosa, E.A.; Silva, D.V.; Santos, J.B.; Pereira, G.A.M. et al. Respostas fisiológicas da mandioca à aplicação de herbicidas. Semina: Ciências Agrárias, v.36, n.2, p.645-656, 2015.

França, A.C.; Freitas, M.A.M.; Fialho, C.M.T.; Silva, A.A.; Reis, M.R.; Galon, L. et al. Crescimento de cultivares de café Arábica submetidos a doses do glyphosate. Planta Daninha, v.28, n.3, p.599-607, 2010.

Genty, B.; Briantais, J.M.; Baker, N.R. The relationship between the quantum yield of photosynthetic electron transport and quenching of chlorophyll fluorescence. Biochimica et Biophysica Acta, v.990, p.87-92, 1989.

Kerr, W.E.; Silva, F.R.; Tchucarramae, B. Pequi (Caryocar brasiliense Camb.). Informações preliminares sobre um pequi sem espinhos no caroço. Revista Brasileira de Fruticultura, v.29, n.1, p.169-171, 2007.

Lu, C.; Wang, B.; Zhang, J. Salinity treatment shows no effects on photosystem II photochemistry, but increases the resistance of photosystem II to heat stress in halophyte Suaeda salsa. Journal Experimental Botany, v.54, p.851-860, 2003.

Macedo, J.F. Pequi: do plantio à mesa. Belo Horizonte: Empresa de Pesquisa Agropecuária de Minas Gerais, Boletim Técnico, 2005, 44 p.

Machado, A.F.L.; Ferreira, L.R.; Santos, L.D.T.; Ferreira, F.A.; Viana, R.G.; Machado, M.S.; Freitas, F.C.L. Eficiência fotossintética e uso da água em plantas de eucalipto pulverizadas com glyphosate. Planta Daninha, v.28, n.2, p.319-327, 2010.

Meschede, D.K.; Velini, E.D.; Carbonari, C.A.; Silva, J.R.M. Alteração fisiológica da cana-deaçúcar pela aplicação de glyphosate e sulfumeturon-methyl. Planta Daninha, v.29, n.2, p.413-419, 2011.

Nandula, V.K.; Reddy, K.N.; Rimando, A.N.; Duke, S.O.; Poston, D.H. Glyphosate- resistant and-susceptible soybean (Glycine max) and canola (Brassica napus) dose response and metabolism relationships with glyphosate. Journal Agricultural Food Chemistry, v.55, n.9, p.3540-3545, 2007.

Pereira, M.R.R.; Rodrigues, A.C.P.; Costa, N.V.; Martins, D.; Klar, A.E.; Silva, M.R. Efeito da deriva de glyphosate sobre algumas características fisiológicas em plantas de eucalipto. Interciencia, v.35, n.4, p.279-283. 2010.

Rascher, U.; Liebig, M.; Luttge, U. Evaluation of instant light-response curves of chlorophyll fluorescence parameters obtained with a 
portable chlorophyll fluorometer on site in the field. Plant, Cell and Environment, v.23, n.12, p.1397-1405. 2000.

Rigoli, R.P.; Fontana, L.C.; Figueredo, S.S.; Noldin, J.A. Resposta de plantas de beterraba (Beta vulgaris) e de cenoura (Daucus carota) à deriva simulada de glyphosate e clomazone. Planta Daninha, v.26, n.2, p.451-456. 2008.

Santos, D.M.M.; Pitelli, R.A.; Banzatto, D.A. Efeitos de herbicidas nos teores de clorofilas de Spirodela punctata. Planta Daninha, v.17, n.2, p.175-182. 1999.

Santos, F.S.; Santos, R.F.; Dias, P.P.; Zanão Jr, L.A.; Tomassoni, F. A cultura do Pequi (Caryocar brasiliense Camb.). Acta Iguazu, v.2, n.3, p.46-57. 2013.

Taiz, L.; Zeiger, E. Fisiologia Vegetal, Porto Alegre: Artmed, 2009, 848p.

Tiburcio, R.A.S.; Ferreira, F.A.; Paes, F.A.S.V.; Melo, C.A.D.; Medeiros, W.N. Crescimento de mudas de clones de eucalipto submetidos à deriva simulada de diferentes herbicidas. Revista Árvore, v.36, n.1, p.65-73. 2012.

Tuffi Santos, L.D.; Sant'anna-Santos, B.F.; Meira, R.M.S.A.; Ferreira, F.A.; Tiburcio, R.A.S. T.; Silva, E.C.F. Micromorfologia foliar na análise da fitotoxidez por glyphosate em Eucalyptus grandis. Planta Daninha, v.27, n.4, p.711-720. 2009.

Vital, R.G. Subdoses de glyphosate e trinexapac-ethyl nas características fisiológicas e de crescimento de plantas de girassol. 2015. 65 f. Dissertação (Mestrado em Ciências Agrárias - Agronomia). IF Goiano Campus-Rio Verde, 2015.

Wagner, J.F.; Merotto Junior, A. Parâmetros fisiológicos e nutricionais de cultivares de soja resistentes ao glifosato em comparação com cultivares isogênicas próximas. Ciência Rural, v.44, n.4, p.393-399. 2014.

Zobiole, L.H.S.; Kremer, R.J.; Oliveira Junior, R.S.; Constantin, J. Glyphosate affects photosynthesis in first and second generation of glyphosate-resistant soybeans. Plant and Soil, v. 336, n.2, p.251-265. $2010 \mathrm{a}$.

Zobiole, L.H.S.; Oliveira Junior, R.S.; Kremer, R.J.; Constantin, J.; Bonato, C.M.; Muniz, A.S. Water use efficiency and photosynthesis of glyphosate-resistant soybean as affected by glyphosate. Pesticide Biochemistry and Physiology, v.97, n.3, p.182-193. 2010 b.

\footnotetext{
photosynthesis in first and second generation of
} 